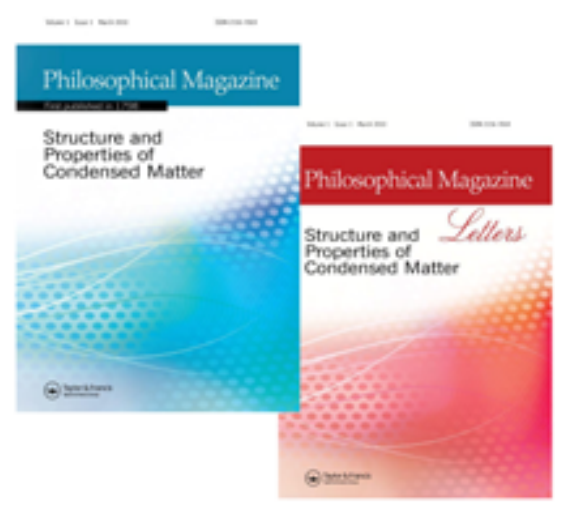

\title{
Topological model of type II deformation twinning in NiTi martensite
}

\begin{tabular}{|c|c|}
\hline Journal: & Philosophical Magazine \& Philosophical Magazine Letters \\
\hline Manuscript ID & TPHM-2018-0456.R1 \\
\hline Journal Selection: & Philosophical Magazine \\
\hline $\begin{array}{l}\text { Date Submitted by the } \\
\text { Author: }\end{array}$ & $\mathrm{n} / \mathrm{a}$ \\
\hline Complete List of Authors: & $\begin{array}{l}\text { Pond, Robert; The University of Exeter, College of Engineering, } \\
\text { Mathematics and Physical Sciences } \\
\text { Hirth, John; Private individual } \\
\text { Knowles, Kevin; University of Cambridge, Department of Materials } \\
\text { Science and Metallurgy; }\end{array}$ \\
\hline Keywords: & $\begin{array}{l}\text { deformation twinning, dislocations, interfacial structures, modelling, } \\
\text { TEM, topological theory of defects }\end{array}$ \\
\hline Keywords (user supplied): & type II twinning, NiTi martensite, disconnections \\
\hline
\end{tabular}

\section{SCHOLARONE" \\ Manuscripts}




\title{
Topological model of type II deformation twinning in NiTi martensite
}

\author{
R. C. Pond ${ }^{\text {a }}$, J.P. Hirth ${ }^{\text {b }}$ and K.M. Knowles ${ }^{c}$
}

${ }^{a}$ College of Engineering, Mathematics and Physical Sciences, University of Exeter, Exeter, EX4 4QF, UK; 'b45 S. Park Centre Ave, Green Valley, AZ 85614, USA; 'Department of Materials Science and Metallurgy, University of Cambridge, 27 Charles Babbage Road, Cambridge CB3 OFS, UK

\begin{abstract}
A topological model of the formation of type II twins in NiTi martensite is presented. Disconnection dipoles are generated initially and expand on the rational $\boldsymbol{k}_{1}=(011)$ plane, subsequently accumulating to form a tilt wall perpendicular to $\boldsymbol{k}_{1}$. Equilibrium occurs when the strain and rotational distortion fields of the constituent defects are equally partitioned between the adjacent crystals. The resultant interface is parallel to the irrational conjugate plane, $\boldsymbol{k}_{2}=(0.721,1, \overline{1})$, consistent with the classical geometric theory of deformation twinning and previously published experimental observations. It is proposed that the formation of type II twins on $\boldsymbol{k}_{2}$ occurs in this manner, rather than formation of type I twins on $\boldsymbol{k}_{1}$, because the disconnections have limited mobility on $\boldsymbol{k}_{1}$ even though they are readily nucleated on this plane. We support this conjecture by showing that the Burgers vector of the defects has a small magnitude, implying easy nucleation, but their motion along $\boldsymbol{k}_{1}$ is accompanied by complicated atomic shuffling.
\end{abstract}

Keywords: type II twinning, NiTi martensite, disconnections, partitioning of distortions

\section{Introduction}

Recently, Pond and Hirth [1] proposed a model for the formation of type II twins, based on the principles of the topological theory of interfacial defects and interface structures. The objective of the present paper is to apply this topological model to NiTi, an important shape-memory alloy [2]. Earlier experimental studies using transmission electron microscopy (TEM) [3] and high resolution TEM [4] confirmed that the observed crystallography of such twins is consistent with the predictions of the classical theory of deformation twinning (see [5] and [6] for reviews).

The crystal structure of NiTi and the crystallography of type II twins are reviewed in Section 2. A brief recapitulation of the topological model is given in Section 3, before this model is applied to NiTi in Section 4. Our conclusions are summarised in Section 5.

For this analysis, we adopt the nomenclature suggested by Frank [7] for the geometrical parameters used in the classical theory, as illustrated in Figure A1 of the Appendix. Conjugate planes in the parent crystal are designated $\boldsymbol{k}_{1}$ and $\boldsymbol{k}_{2}$, and are mutually inclined by the angle $2 \phi$. Following a plane-strain, volume conserving lattice deformation, these planes are rotated but undistorted, becoming the planes labelled $\boldsymbol{k}_{1}^{\prime}$ and $\boldsymbol{k}_{2}^{\prime}$; these are 
also mutually inclined by $2 \phi$. The directions lying in the four planes, $\boldsymbol{k}_{1}, \boldsymbol{k}_{2}, \boldsymbol{k}_{1}^{\prime}$ and $\boldsymbol{k}_{2}^{\prime}$, and also in the plane of shear, are designated $\boldsymbol{\gamma}_{1}, \boldsymbol{\gamma}_{2}, \boldsymbol{\gamma}_{1}^{\prime}$ and $\boldsymbol{\gamma}_{2}^{\prime}$, respectively. We define another angle, $\alpha=\pi / 2-2 \phi$. If the lattice deformation is supplemented by an anticlockwise rotation about the unit normal to the plane of shear, $\boldsymbol{n}_{s}$ (this vector points out of the page in Figure A1), $\boldsymbol{k}_{1}^{\prime}$ is brought into coincidence with $\boldsymbol{k}_{1}$, and $\boldsymbol{\gamma}_{1}^{\prime}$ with $\boldsymbol{\gamma}_{1}$, Figure A1(a). Twins with this crystallography which are observed experimentally are designated $\boldsymbol{K}_{1}, \boldsymbol{\eta}_{1}$, exhibiting the (invariant) twin plane $\boldsymbol{K}_{1}=\boldsymbol{k}_{1}$ and twinning direction, $\boldsymbol{\eta}_{1}=\boldsymbol{\gamma}_{1}$. If the rotation by $\alpha$ about $\boldsymbol{n}_{s}$ is clockwise, Figure A1(b), the conjugate twin would be observed, with, $\boldsymbol{K}_{1}=\boldsymbol{k}_{2}$ and $\boldsymbol{\eta}_{1}=\boldsymbol{\gamma}_{2}$ . The property pointed out by Cahn [8] is of particular significance in the present context: when the plane of shear is irrational, one conjugate is type I, i.e. $\boldsymbol{k}_{1}$ is rational and $\boldsymbol{\gamma}_{1}$ is irrational, while the other is type II, i.e. $\boldsymbol{k}_{2}$ is irrational and $\boldsymbol{\gamma}_{2}$ is rational. In the classical geometrical model of deformation twinning $(\mathrm{CM})$, both conjugates exhibit the same value of engineering shear, which can be expressed as $s=2 \tan \alpha[5]$.

\section{Crystallography of NiTi}

Following the martensitic transformation from the high-temperature cubic form, NiTi exhibits monoclinic symmetry. Michal and Sinclair [9] used X-ray diffraction to establish the crystal structure. This is illustrated by the unit cell in Figure 1 in which planes and directions are indexed with respect to the conventional monoclinic cell with the unique axis parallel to [010]. In this setting, the lattice parameters are: $a_{m}=0.2885 \mathrm{~nm}, b_{m}=0.4120 \mathrm{~nm}, c_{m}$ $=0.4622 \mathrm{~nm}$, and $\beta_{m}=96.8^{\circ}$. The crystal exhibits the space group $P 2_{1} / \mathrm{m}$. The atomic motif is comprised of four atoms: Ti at $0,0,0$ and $0.055,0.5,0.558$, and $\mathrm{Ni}$ at $0.580,0,0.472$ and $0.475,0.5,0.086$.

Using the expressions derived by Jaswon and Dove [10], Knowles and Smith [3] determined the characteristic parameters according to the CM theory, shown in Table 1. Since the angle $2 \phi=82.02^{\circ}$, the rotation angle is $\alpha=7.98^{\circ}$, and hence the shear is $s=0.280$. The plane of shear, $\boldsymbol{P}_{s}$, is $(\overline{1}, 0.788, \overline{0.788})$, which is clearly irrational and contains the directions $\gamma_{1}$ and $\boldsymbol{\gamma}_{2}$. Figure 2(a) is a TEM image from the work of Knowles and Smith [3] showing an array of type II twins in NiTi viewed edge on to the electron beam. The accompanying diffraction pattern, Figure $2(\mathrm{~b})$, was recorded with the electron beam oriented along $\gamma_{2}$. Since this direction is the two-fold twinning axis interrelating the two crystals, twin and parent reflections superpose. In addition, the twin plane orientation was measured to be $10 \pm 2^{\circ}$ away from $(11 \overline{1})_{\mu}$ towards $(01 \overline{1})_{\mu}$, in good agreement with the calculated value of $10.07^{\circ}$. Thus, the observed twin is consistent with being type II, with $\boldsymbol{K}_{1}$ ${ }^{,} \boldsymbol{\eta}_{1}=\boldsymbol{k}_{2}, \boldsymbol{\gamma}_{2}$.

\section{Topological Model}

Whereas the mechanism of type I and compound twinning is understood in terms of the generation and motion of disconnections along rational interfaces, this mechanism cannot occur on irrational twin planes. Disconnections have both dislocation character, represented by their Burgers vector, $\boldsymbol{b}$, and step character, $h$, equal to some integer multiple of the rational plane spacing [11]. For irrational twin boundaries, no step height can 
be defined, and hence no disconnection can be characterised. Pond and Hirth [1] have reconciled this dichotomy. This is illustrated in Figure 3 for cases where the plane of shear is irrational. Type I conjugate twins can form by nucleation and expansion of disconnection dipoles on the $\boldsymbol{k}_{1}$ plane, which is referred to here as the disconnection glide plane: these disconnection dipole defects accumulate, forming the tips of lenticular twins, as has been recognised for many years $[5,6]$. Such motion of disconnections produces the engineering shear $\gamma=b / h$. For specific disconnections $\gamma=s$, so the mechanistic model corresponds directly to the simple shear obtained by the classical geometric theory.

The formation of type II twins is also illustrated in Figure 3. Here, the disconnections described above are again generated on the $\boldsymbol{k}_{1}$ glide plane, but accumulate in a different manner, forming a tilt wall perpendicular to $\boldsymbol{k}_{1}$. After partitioning of the strains and rotational distortions of the defects between the adjacent crystals, a symmetrical tilt boundary is formed on the $\boldsymbol{k}_{2}$ plane. The misorientation can be described by, for example, the axis/angle pair, $\boldsymbol{n}_{s} / 2 \alpha$, or $\boldsymbol{\gamma}_{2} / \pi$. In this way, the interface formed corresponds to the type II conjugate twin on the invariant $\boldsymbol{k}_{2}$ plane. However, while the overall deformation is equal to a simple shear of magnitude $s$ on $\boldsymbol{k}_{2}$, the mechanism of formation involves both shear on $\boldsymbol{k}_{1}$ and accommodational relaxation. Hence, it does not correspond directly with the classical model.

Currently, the final microstructure of such a type II interface is not known. Although all strains are removed at some distance from the interface, the near field might produce a non-periodic arrangement of micro-facets, for example. Away from the broad $\boldsymbol{k}_{2}$ faces of a type II twin, strains arise around the tips of lenticular forms, with long-range fields analogous to type I cases.

As outlined above, the broad faces of type II twins cannot advance by motion of disconnections along them, and the micro-mechanism of this process is not currently understood. However, application of the phenomenological treatment of Read and Shockley [12] shows that the shear coupled with migration is equal to $s=2 \tan \alpha$, consistent with the classical model.

According to the model of Pond and Hirth [1], whether the type I or type II conjugate arises is determined on a competitive basis, consistent with the experimental finding that either the type I or type II conjugate is usually observed exclusively, both being observed simultaneously only rarely, such as in $\alpha$-uranium $[8,13]$. We propose that type I twins form preferentially when the ratio $\dot{G} / \dot{N}$ is sufficiently large, and type II for lower values. $\dot{G}$ represents the twin growth rate, which is dominated by the disconnection mobility on the $\boldsymbol{k}_{1}$ glide plane, and $\dot{N}$ is the nucleation rate of disconnection pairs on this plane. Rigorous determination of $\dot{G}$ requires sophisticated atomic-scale simulations, currently being developed [14]. For present purposes, we adopt a simpler estimation based on the atomic shuffling accompanying the motion of a disconnection as it sweeps through one period of the $\boldsymbol{k}_{1}$ glide plane [15]. The larger the number of atoms shuffling in this process, and the more complex their trajectories, the lower $\dot{G}$ is expected to be. Accurate values of $\dot{N}$ also 
require sophisticated simulations, [16], but here we simply use $b^{2}$ as a guide, reflecting the elastic energy involved in nucleation.

\section{Topological Model for NiTi}

We begin by finding the Burgers vectors of candidate (perfect) disconnections on the $\boldsymbol{k}_{1}=(011)$ glide plane. These correspond to differences between translation vectors, one from the (upper) twin crystal, designated $\boldsymbol{t}(\lambda)$, and one from the (lower\} parent crystal, designated $\boldsymbol{t}(\mu)$ [17]. Thus, expressed using the parent crystal's coordinate frame, we have

$$
\boldsymbol{b}=\boldsymbol{P}^{-1} \boldsymbol{t}(\lambda)-\boldsymbol{t}(\mu),
$$

where $\boldsymbol{P}^{-1}$ represents a coordinate transformation from the $\lambda$ to the $\mu$ frame. A convenient choice for $\boldsymbol{P}$ is the rotation $\boldsymbol{n}_{\boldsymbol{k}_{1}} / \pi$ : this describes the type I twin misorientation for centrosymmetric crystals. An alternative graphical representation is as a difference vector in the dichromatic pattern [17], i.e. the pattern obtained by the interpenetrating $\lambda$ and $\mu$ lattices.

The step height is given by

$$
h=\boldsymbol{n}_{\boldsymbol{k}_{1}} \cdot \boldsymbol{P}^{-1} \boldsymbol{t}(\lambda)=\boldsymbol{n}_{\boldsymbol{k}_{1}} \cdot \boldsymbol{t}(\mu)
$$

and this must correspond to $h=p d_{\boldsymbol{k}_{1}}$, where $p$ is an integer and $d_{\boldsymbol{k}_{1}}$ is the interplanar spacing of the $\boldsymbol{k}_{1}$ lattice planes. To be consistent with the RH/FS rule [18], the line direction of the disconnection is taken to be $\boldsymbol{n}_{s}$ (out of the page in Figure A1).

For $\mathrm{NiTi}$, the smallest magnitude Burgers vector is obtained with $\boldsymbol{t}(\mu)=[001]_{\mu}$ and $\boldsymbol{t}$ $(\lambda)=[010]_{\lambda}$, giving $\boldsymbol{b}=[0.169,0.108, \overline{0.108}]$ which is parallel to $\boldsymbol{\gamma}_{1}$ with magnitude 0.086 $\mathrm{nm}$. This magnitude is sufficiently small for homogeneous nucleation of disconnection loops at the stresses typically reached during nucleation [5], and implies a reasonable value of $\dot{N}$. The step height of this defect is $h=d_{(011)}=0.307 \mathrm{~nm}$ (i.e., $p=1$ in this case). The engineering shear, $\gamma$, produced by motion of this defect along $\boldsymbol{k}_{1}$ is $b / h=0.280$, which is the same as the classical value, $s$. A graphical representation of $\boldsymbol{b}$ is shown in Figure 4(a) which shows selected vectors in the dichromatic pattern, including the coincident translation vectors in the $\boldsymbol{k}_{1}$ plane, $[0 \overline{1} 1]_{\mu}$ and $[100]_{\mu}$, and the vectors $\boldsymbol{t}(\lambda)$ and $\boldsymbol{t}(\mu)$ projected onto this plane. It can be seen that $\boldsymbol{b}$ is parallel to $\boldsymbol{\gamma}_{1}$, and also that $\boldsymbol{n}_{s}$ is perpendicular to $\gamma_{1}$. The projection onto the plane of shear is shown in Figure 4(b): this illustrates that $h=d_{(011)}$, and also the classical vectors $\boldsymbol{\gamma}_{2}$ and $\boldsymbol{\gamma}_{2}^{\prime}$ which are mutually inclined by $2 \alpha$.

To assess the mobility of this disconnection, we construct the dichromatic complex which is obtained by envisioning the $\lambda$ and $\mu$ crystal structures to interpenetrate $[1,15]$. From this complex we establish the elementary transformation volume (ETV) which contains the $N$ distinct atoms that must be relocated from $\mu$ sites to $\lambda$ sites when the disconnection sweeps one period of the $\boldsymbol{k}_{1}$ interface [16], Figure 5: the $\mu$ sites are located in the cell defined by $[100]_{\mu},[0 \overline{1} 1]_{\mu},[001]_{\mu}$, and the $\lambda$ sites in the cell defined by $[\overline{100}]_{\lambda}[01 \overline{1}]_{\lambda}$, $[010]_{\lambda}$. In the present case, $N=4$, i.e. two Ti and two Ni sites. In Figure 5, the four $\mu$ sites are shown projected onto the $\boldsymbol{k}_{1}$ plane, and the set of corresponding $\lambda$ sites is obtained by 
operating through the origin, $\mathrm{O}$, the 2-fold twinning operation which is perpendicular to the plane, and, when necessary, adding translation vectors in this plane. All $N$ atoms are displaced by $\boldsymbol{b}$, and any additional shuffles are labelled $\boldsymbol{s}_{i}, i=1$ to $N$; therefore the total displacements are $\boldsymbol{u}_{i}=\boldsymbol{b}+\boldsymbol{s}_{i}$. In reality, the set $\boldsymbol{u}_{i}$ depends on the relative position of the two crystals, $\boldsymbol{p}$, but, since this is unknown in the present case, we assume the crystals are in coincidence, so that $\boldsymbol{p}=0$ for this reference state. In the NiTi case $N=4$ : two Ni atoms are located near the first (022) plane at height $h / 2$ above the glide plane, and two Ti atoms near the second (022) plane at height $h$. Their displacements, $\boldsymbol{u}_{i}$, are illustrated graphically in Figure 5, and the corresponding shuffles are listed in Table 2.

The shuffles listed in Table 2 are quite complex: in the (011) plane, one Ti atom in the parent is sheared into the correct position in the twin by passage of the disconnection, while the other shuffles parallel to the glide plane by $0.048 \mathrm{~nm}$ but inclined steeply to $\boldsymbol{\eta}_{1}$. Both $\mathrm{Ni}$ atoms in the (022) plane shuffle parallel to this plane by $0.055 \mathrm{~nm}$, and by equal but opposite shuffle components of $0.035 \mathrm{~nm}$ perpendicular to the plane; in addition, their overall shuffles are inclined by $130.7^{\circ}$ to $\boldsymbol{\eta}_{1}$. Consequently, we anticipate that $\dot{G}$ is likely to be relatively low. Thus, the figure of merit, $\dot{G} / \dot{N}$, is also likely to be low in this case, so that type II twinning is expected, consistent with the observations [3, 4] of $\boldsymbol{K}_{1}, \boldsymbol{\eta}_{1}=\boldsymbol{k}_{2}, \boldsymbol{\gamma}_{2}=$ $(0.721,1, \overline{1})[011]$ twinning. The limited mobility surmised here can be contrasted with two other cases where $N=4$ but with higher mobility. Both examples are in compound twins where the conjugates are crystallographically equivalent. In NiMnGa, all four atoms are coplanar in $\boldsymbol{K}_{1}$ and move without shuffles, i.e. $\boldsymbol{s}_{i}=0$ [19]. The other example where $\dot{G} / \dot{N}$ might be above the transition value is the twinning disconnection in (1012) twins in hcp metals. Although the shuffles in this case have similar magnitudes to those in NiTi, their directions are much more simply related to the twinning parameters, two being parallel and anti-parallel to $\boldsymbol{n}_{\boldsymbol{k}_{1}}$ and two being parallel and anti-parallel to $\boldsymbol{\eta}_{1}$ [16]. By contrast, three of the four shuffle directions in NiTi are steeply inclined to $\boldsymbol{\eta}_{1}$, Table 2.

When the disconnections in NiTi accumulate into a tilt wall they lose their step character. Moreover, as equilibrium is reached, their strains and rotational distortions are partitioned equally (in the homogeneous isotropic elastic approximation) between the nascent twin and parent crystals. Schematically, each disconnection can be regarded as having been partitioned equally, i.e. b/2 in each crystal, Figure 6 . Under these circumstances there is no long-range strain since the component of $\boldsymbol{b}$ parallel to the final interface is zero, and a misorientation of $2 \alpha$ develops about $\boldsymbol{n}_{s}$. In the present case 2 $\alpha=15.96^{\circ}$; however, the interface should not be regarded as a low-angle grain boundary because there are no regions of distorted single crystal between the constituent defects which are separated by $h / \cos \alpha=0.310 \mathrm{~nm}$. It is also evident from Figure 6 that $\tan \alpha=$ $b / 2 h$, which is consistent with the classical relation $s=2 \tan \alpha$. An equivalent description of the misorientation is obtained by combining the initial value, $\boldsymbol{n}_{k_{1}} / \pi$, with the additional value, $\boldsymbol{n}_{s} / 2 \alpha$, giving a final value of $\gamma_{2} / \pi$, which is the classical description of a type II twin.

\section{Summary and Discussion}


The model of Pond and Hirth [1] has been applied to NiTi. The irrational twin interface, misorientation and twinning direction are found to be consistent with experimental observations $[3,4]$ and the geometrical predictions of the classical theory $[5$, 6]. According to the model [1], the mechanism of formation of type II twins involves both shear and accommodation by relaxation, although the overall distortion is a simple shear on the twin plane, consistent with the classical shear, $s$. The shearing mechanism is the generation and glide of disconnections with Burgers vector $\boldsymbol{b}$ and step height $h$ on the conjugate rational $\boldsymbol{k}_{1}$ plane. If these disconnections have limited mobility leading to a low value of the figure of merit $\dot{G} / \dot{N}$, the disconnections assemble into a symmetrical tilt boundary exhibiting type II crystallography. $\dot{G}$ and $\dot{N}$ were estimated for NiTi, the former by $b^{2}$, and the latter by the complexity of atomic shuffling accompanying disconnection glide. In this way, the ratio $\dot{G} / \dot{N}$ was surmised to be relatively modest, consistent with the observed formation of type II twins $[3,4]$. The misorientation of the symmetrical tilt boundary in NiTi can be described as $\boldsymbol{n}_{s} / 2 \alpha$, where $2 \alpha=15.96^{\circ}$, or equivalently as $\boldsymbol{\eta}_{1} / \pi$.

Two aspects of the proposed model require further development. One is a more precise determination of $\dot{G}$ and $\dot{N}$; atomic-scale simulations are providing insights into these properties $[14,15,16]$. The second aspect is the micro-mechanism by which the type II interface migrates. As a preliminary step, an understanding of the micro-structure of such interfaces is needed. Perhaps, for example, the equilibrium boundary exhibits a nonperiodic arrangement of rational micro-facets. As is confirmed by Figure $2 b$, all planes in the $\boldsymbol{\eta}_{1}=[011]$ zone are common, so micro-facet combinations could form an interface with overall orientation parallel to $\boldsymbol{K}_{1}=\boldsymbol{k}_{2}$ : for example, Knowles [4] has suggested a combination of $(01 \overline{1})$ and $(11 \overline{1})$ facets. Although these facets have rational indices in each crystal, it is emphasized that the tilt axis, $\boldsymbol{\eta}_{1}$, is the only coincident rational direction in the facets.

Detwinning upon unloading is also important for NiTi in shape-memory applications [20]. The reverse twinning is subject to Bauschinger constraints, i.e., there is some pinning and relaxation of the forward twin so that the backward process is not thermodynamically reversible. One possible mechanism of type II detwinning is the reverse of the twinning mechanism described above. Here, pairs of dislocations, one originating from each of the opposite tilt walls, would revert to being disconnections and annihilate on terminal $\boldsymbol{k}_{1}$ planes as the twin recedes longitudinally. In related work, Braisaz et al. [21] have studied a similar mechanism at the blunt ends of $\{10 \overline{1} 2\}$ twins in $\mathrm{Zn}$ : in that compound twinning case, the blunt end facets and the lateral twin planes are crystallographically equivalent rational conjugate planes.

\section{Acknowledgement}

We dedicate this paper to the memory of Alan G Crocker (1935-2018) who made seminal contributions to the mathematical theory of deformation twinning, the mechanism of twin growth by defect motion, and simulations of interfacial structure. 


\section{Appendix Linear and non-linear models of twinning}

Figure $\mathrm{A} 1(\mathrm{a})$ is a diagrammatic representation of the $\mathrm{CM}[5,6]$ in which the deformation to form a type I twin is produced by combining a volume conserving planestrain pure shear expressed with respect to the principal strain axes, $x_{1}, x_{2}, x_{3}$, with an anticlockwise rotation by the angle $\alpha$ about $x_{1}$ which brings $\boldsymbol{k}_{1}^{\prime}$ into coincidence with $\boldsymbol{k}_{1}$. Similarly, a type II twin is formed when the rotation is clockwise, figure A1(b), bringing $\boldsymbol{k}_{2}^{\prime}$ into coincidence with $\boldsymbol{k}_{2}$. Phenomenologically, the overall deformation in both cases can be expressed as an engineering shear with magnitude, $s$, on the invariant plane. From the geometry of the figures, this shear can either be expressed as $s=2 \tan \alpha$, corresponding to QP in the figures, or as $s=\tan \beta[5,6]$. In Appendix A of reference [1], a mathematical formulation of the $\mathrm{CM}$ was presented which obtained the same results. However, as pointed out there, that formulation is only valid to linear order, i.e. for small angles when $2 \alpha=\beta$ : this limitation is a consequence of the approximations inherent in the method of coordinate transformation employed, i.e., the transformed strain tensor is more complicated in the nonlinear case [22]. By contrast, the TM and its mathematical formulation are valid to both linear and non-linear order, justifying its use in reference [1] and this work.

To compare the geometrical CM with the mechanistic TM, we use engineering strain coordinates in order to avoid the coordinate transformation issues. The mechanistic interpretation of the displacement, $\boldsymbol{s}$, at unit distance from the twin plane according to the $\mathrm{CM}$ is illustrated in figure $\mathrm{A} 2(\mathrm{a})$, emphasizing the twin symmetry, and (b) the engineering strain, $\gamma$. For the type I twin, the rational direction $\boldsymbol{\gamma}_{2}$ is selected from the crystal lattice, and the angle $\alpha$ follows from the twin parameters. In the TM, the Burgers vector of the active disconnection, $\boldsymbol{b}$, is either obtained analytically from equation (1) and the step height, $h$, from equation (2), or graphically from the dichromatic pattern. In Sections 3 and 4 we demonstrated that $s=b / h$. Thus, for type I twins, whether one determines $\gamma$ using the $\mathrm{CM}$ or TM, the same geometry and magnitude of shear are obtained, i.e. we can write,

$$
\gamma=s=b / h=\tan \beta=2 \tan \alpha .
$$

In other words, the TM agrees to non-linear order for type I twins with the geometry of the CM.

As described in Section 3, the non-linear TM for type II twinning explicitly includes partitioning. Moreover, figure 6 illustrates that the geometry of the equilibrium interface remains consistent with equation (A1), and hence with the geometry of figure $A 1(b)$. This parallel between the TM and $\mathrm{CM}$ is further emphasized by modifying figure $\mathrm{A} 1$ (b) to show the two deformations producing the displacements in each crystal, $\boldsymbol{u}_{\mu}$ and $\boldsymbol{u}_{\lambda}$, both of which have magnitude $s / 2$.

According to the $\mathrm{CM}$ [5], the same magnitude of $s$ is obtained for both conjugates, and, as shown above, the same is true for the TM. Supporting the latter result, the type I twin could be formed alternatively by a hypothetical process, figure A3. Beginning with a plane $\boldsymbol{k}_{2}^{0}$ in a notional single crystal, the twin is formed by a shear displacement $s / 2$, 
subtending the angle $-\alpha$ while the matrix is produced by the shear displacement $s / 2$, subtending the angle $+\alpha$. Thus, with partitioning, the TM treatments of type I and II give identical results, agreeing with the $\mathrm{CM}$ geometry, figure $\mathrm{A} 1$. Whereas the partitioning in figure $A 3$ is shown as a hypothetical two-stage process, the actual mechanism would be direct local rearrangement into the partitioned configuration as disconnections accumulate [1]. That is, local non-linear rearrangements at the disconnection cores produce an interface agreeing with macroscopic twin symmetry, analogous to the mechanism of tilt wall formation [23]. A corollary for compound twins is that, without ancillary information, one cannot distinguish whether such a twin formed by disconnection glide along the $\boldsymbol{K}_{1}$ plane or by disconnection glide on $\boldsymbol{K}_{2}$ accompanied by rotational accommodation.

We have shown above that the interface plane and shear predicted by the TM agree with the CM geometry, but that the mathematical formulation of the $\mathrm{CM}$ in [1] is only valid to linear order. Also, to nonlinear order, incompatibilities arise at the interface unless partitioning is included [24], which is not the case for the CM. Since the mathematical treatment of the phenomenological theory of martensite crystallography (PTMC) [25] is an extension of the CM formalism [5, 6], we outline the consequential limitations of a linear treatment. In the PTMC the Bain strain may not be volume conserving and an additional lattice-invariant distortion is required. Elsewhere we have shown that these additional factors, related to partitioning, lead to discrepancies between the habit planes predicted using the PTMC and the TM [26], although these are generally small [27].

\section{References}

[1] R.C. Pond and J.P. Hirth, Topological model of type /l deformation twinning, Acta Mater. 151 (2018), pp. 229-242.

[2] K. Otsuka and C.M. Wayman, (eds.), Shape Memory Materials, Cambridge University Press, Cambridge, 1998.

[3] K.M. Knowles and D.A. Smith, The crystallography of the martensitic transformation in equiatomic nickel-titanium, Acta Metall. 29 (1981), pp. 101-110.

[4] K.M. Knowles, A high-resolution electron microscope study of nickel-titanium martensite, Phil. Mag. A 45 (1982), pp. 357-370.

[5] J.W. Christian and S. Mahajan, Deformation twinning, Prog. Mater. Sci. 39, 1995, pp. 1157.

[6]. O.B.M. Hardouin Duparc, A review of some elements for the history of mechanical twinning centred on its German origins until Otto Mügge's $\boldsymbol{k}_{1}$ and $\boldsymbol{k}_{2}$ invariant plane notation, J. Mater. Sci. 52 (2017), pp. 4182-4196. 
[7] F.C. Frank, A note on twinning in alpha-uranium, Acta Metall. 1 (1953), pp. 71-74.

[8] R.W. Cahn, Plastic deformation of alpha-uranium; twinning and slip, Acta Metall. 1 (1953), pp. 49-70.

[9] G.M. Michal and R. Sinclair, The structure of TiNi martensite, Acta Cryst. B37 (1981), pp. 1803-1807.

[10] M.A. Jaswon and D.B. Dove, The crystallography of deformation twinning, Acta Cryst. 13 (1960), pp. 232-240.

[11] J.P. Hirth and R.C. Pond, Steps, dislocations and disconnections as interface defects relating to structure and phase transformations, Acta Mater. 44 (1996), pp.4749-4763.

[12] W.T. Read and W. Shockley, Dislocation models of crystal grain boundaries, Phys. Rev. 78 (1950), pp. 275-289.

[13] R.J. McCabe, L. Capolungo, P.E. Marshall, C.N. Cady and C.N. Tomé, Deformation of wrought uranium: Experiments and modelling, Acta Mater. 58 (2010), pp. 5447-5459.

[14] E. Martínez, L. Capolungo, and C.N. Tomé, Atomistic analysis of the $\{10 \overline{1} 2\}$ twin stability and growth in $\alpha$-Ti, Phys. Rev. Mater. 2 (2018), pp. 083603-1-083603-10.

[15] R.C. Pond, J.P. Hirth, A. Serra and D.J. Bacon, Atomic displacements accompanying deformation twinning: shears and shuffles, Mater. Res. Lett. 4 (2016), pp. 185-190.

[16] H.A. Khater, A. Serra, and R.C. Pond, Atomic shearing and shuffling accompanying the motion of twinning disconnections in Zirconium, Phil. Mag. 93 (2013), pp. 1279-1298.

[17] R.C. Pond, Line defects in interfaces, in Dislocations in Solids 8, F.R.N. Nabarro, ed., North-Holland, Amsterdam, 1989, pp. 1-66.

[18] P.M. Anderson, J.P. Hirth, and J. Lothe, The Theory of Dislocations, 3rd ed. Cambridge University Press, Cambridge, 2017.

[19] R.C. Pond, B. Muntifering, and P. Müllner, Deformation twinning in $\mathrm{Ni}_{2} \mathrm{MnGa}$, Acta Mater. 60 (2012), pp. 3976-3984.

[20] K. Otsuka and X. Ren, Physical metallurgy of Ti国i-based shape memory alloys, Prog. Mater. Sci. 50 (2005), pp. 511-678.

[21] T. Braisaz, P. Ruterana and G. Nouet, Twin tip defects related to the nucleation and growth mechanisms of the twin (1012) in zinc characterized by high-resolution electron microscopy, Phil. Mag. A 76 (1997), pp. 63-84.

[22]. J.D. Clayton, Nonlinear Mechanics of Crystals, Springer publishing, New York, 2011.

[23]. J. P. Hirth, R. C. Pond and J. Lothe, Disconnections in Tilt Walls, Acta Mater., 54 (2006), pp. 4237-45.

[24]. J. P. Hirth, J. Wang and C.N. Tomé, Disconnections and other defects associated with twin interfaces, Prog. Mater. Sci., 83 (2016), pp. 417-471. 
[25]. C.M. Wayman, Introduction to the crystallography of martensite transformations, Macmillan, New York, 1964.

[26]. J.P. Hirth, R.C. Pond, R.G. Hoagland, X.-Y. Liu, and J. Wang, Interface defects, reference spaces and the Frank-Bilby equation, Prog. Mater. Sci., 58 (2013), pp. 749-823.

[27]. R.C. Pond, X. Ma, Y.W. Chai, and J.P. Hirth, Topological modelling of martensitic transformations, in Dislocations in solids, 13, F.R.N. Nabarro and J.P. Hirth, eds., Elsevier, Amsterdam, 2007, pp. 225-262.

Table 1. Crystallographic parameters for type II twinning in NiTi predicted using the classical theory of deformation twinning [3].

\begin{tabular}{|c|c|}
\hline $\boldsymbol{K}_{1}=\boldsymbol{k}_{2}$ & $(0.721,1, \overline{1})$ \\
\hline $\boldsymbol{\eta}_{1}=\boldsymbol{\gamma}_{2}$ & {$[011]$} \\
\hline $\boldsymbol{K}_{2}=\boldsymbol{k}_{1}$ & $(011)$ \\
\hline $\boldsymbol{\eta}_{2}=\boldsymbol{\gamma}_{1}$ & {$[1,0.636, \overline{0.636}]$} \\
\hline $2 \phi$ & $82.02^{\circ}$ \\
\hline$S$ & 0.280 \\
\hline
\end{tabular}

Table 2. Atomic shuffles, $\boldsymbol{s}_{i}$, and the magnitudes of their components parallel, $\left|s_{i}^{\|}\right|$, and perpendicular, $\left|s_{i}^{\perp}\right|$, to the disconnection glide plane are listed. $\theta$ is the angle between $\boldsymbol{s}_{i}$ and $\boldsymbol{\eta}_{1}$. Shuffles 1 and 2 relate to Ti atoms, and 3 and 4 to $\mathrm{Ni}$ atoms.

\begin{tabular}{|c|c|c|c|c|c|}
\hline$i$ & $\boldsymbol{s}_{i}$ & $\left|\boldsymbol{s}_{i}\right| n m$ & $\left|s_{i}\right| \mathrm{nm}$ & $\left|s_{i}{ }^{\perp}\right| n m$ & $\theta^{\circ}$ \\
\hline 1 & {$[0,0,0]$} & 0 & 0 & 0 & 0 \\
\hline 2 & {$[\overline{0.105}, 0.064, \overline{0.064}]$} & 0.048 & 0.048 & 0 & 73.6 \\
\hline 3 & {$[\overline{0.125}, 0.041,0.072]$} & 0.055 & 0.042 & 0.035 & 130.7 \\
\hline 4 & {$[\overline{0.144} \overline{0.085, \overline{0.029}]}$} & 0.055 & 0.042 & 0.035 & 130.7 \\
\hline
\end{tabular}




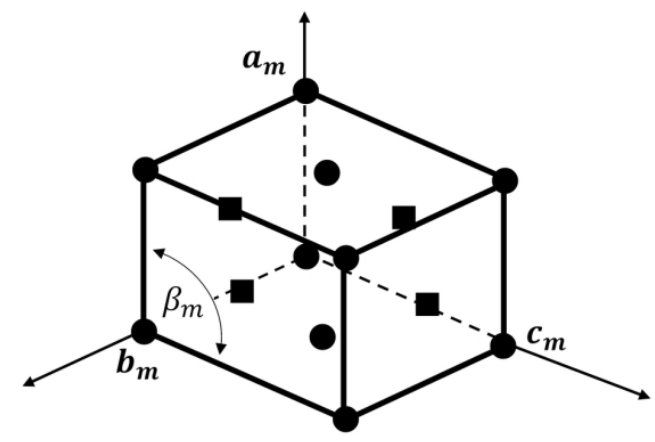

Figure 1. Unit cell of monoclinic NiTi, after [9], showing Ti sites as circles and Ni sites as squares. The actual atomic sites are displaced slightly from these nominal edge and face-centring positions, as described in Section 2.

$159 \times 83 \mathrm{~mm}(300 \times 300$ DPI $)$ 

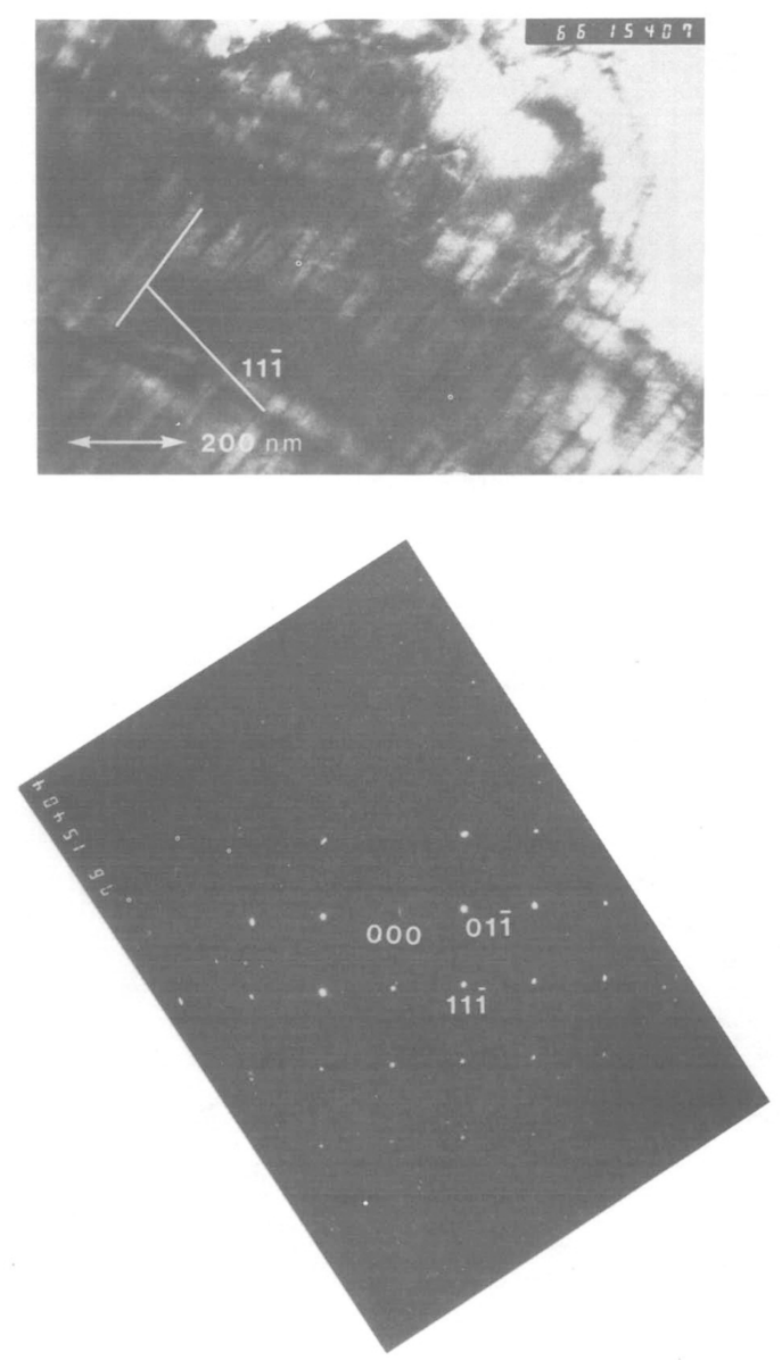

Figure 2. (a) TEM image of edge-on type II interfaces in NiTi with the beam directed along $\boldsymbol{\eta}_{1}=[011]$. (b) The corresponding diffraction pattern shows that all planes in this zone are common, and that the twin plane is oriented $10 \pm 2^{\circ}$ away from $(11 \overline{1})_{\mu}$ towards $(01 \overline{1})_{\mu}$. Reproduced with permission from Elsevier.

\section{$157 \times 233 \mathrm{~mm}(300 \times 300 \mathrm{DPI})$}




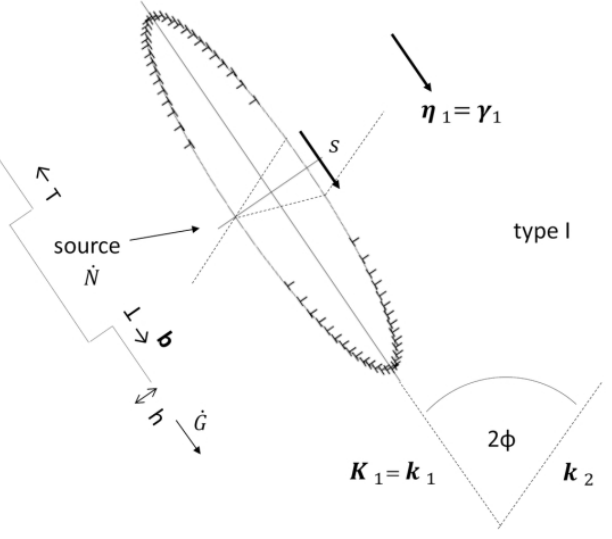

(a)

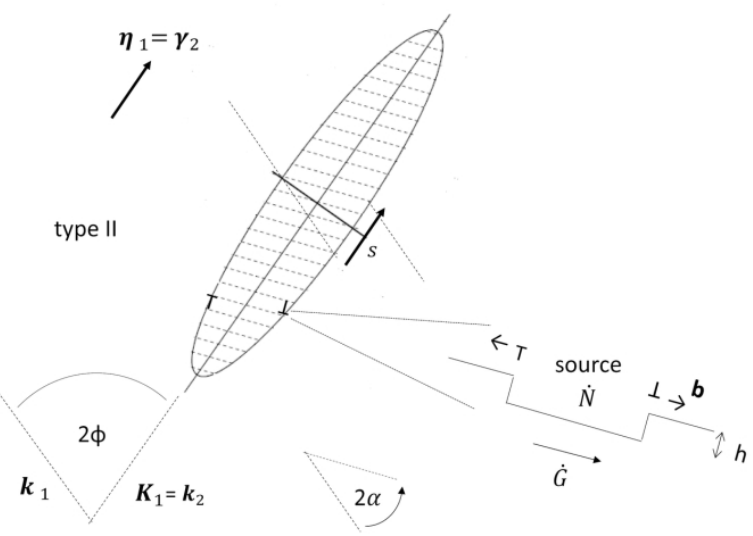

(b)

Figure 3. Schematic illustration of the topological model for the formation of type I (a) and type II twins (b). The plane of shear is parallel to the page, and is irrational. Dipole sources operate on the disconnection glide plane, $\boldsymbol{k}_{1}$, in both cases, as indicated by the inset sketches. After relaxation, the $\boldsymbol{k}_{1}$ planes within the type II twin are rotated by $2 \alpha$ about $\boldsymbol{n}_{s}$ from their original orientation. $167 \times 219 \mathrm{~mm}(300 \times 300 \mathrm{DPI})$ 


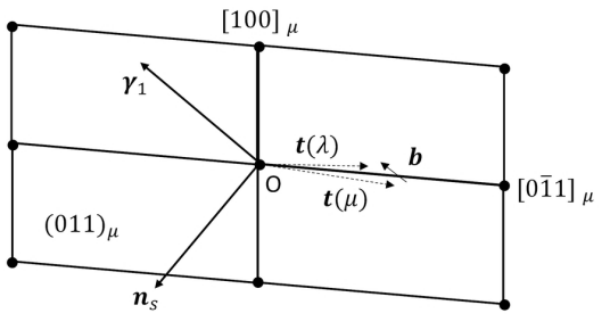

(a)

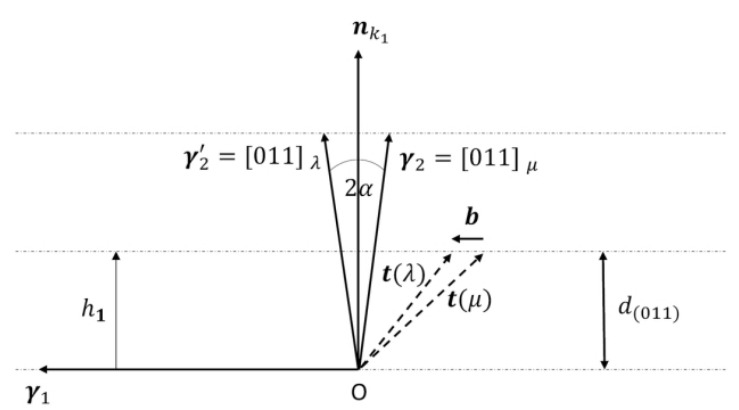

(b)

Figure 4. (a) Selected vectors of the dichromatic pattern projected onto $\boldsymbol{k}_{1}$, and (b) projection onto the plane of shear, $\boldsymbol{P}_{s}$. The translation vectors $\boldsymbol{t}(\mu)=[001]_{\mu}$ and $\boldsymbol{t}(\lambda)=[010]_{\lambda}$ are inclined to both $\boldsymbol{k}_{1}$ and $\boldsymbol{P}_{S}$ : their difference is equal to $\boldsymbol{b}$.

$162 \times 150 \mathrm{~mm}(300 \times 300 \mathrm{DPI})$ 
$165 \times 78 \mathrm{~mm}(300 \times 300 \mathrm{DPI})$ 


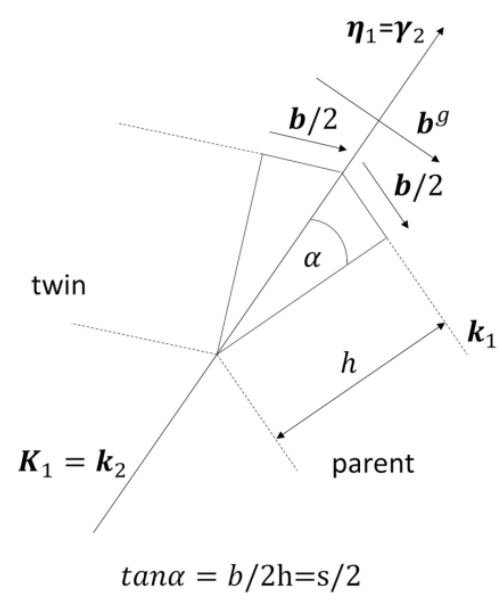

(a)

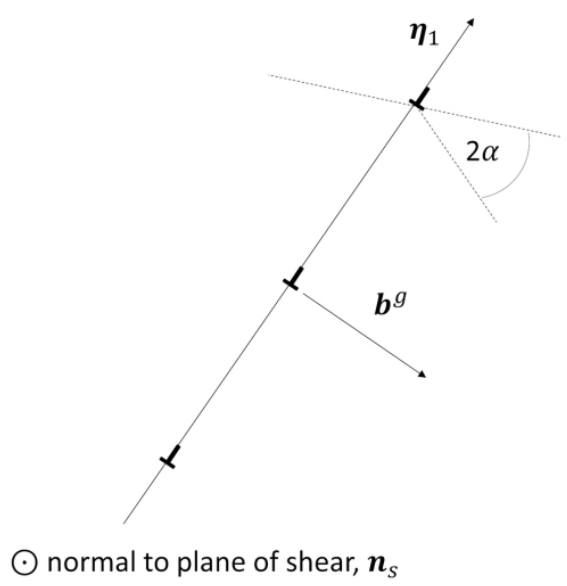

(b)

Figure 6. Schematic illustration of the symmetrical tilt boundary formed on $\boldsymbol{k}_{2}$ after partitioning of the strains and rotational distortions of the constituent defects, (a). Schematic illustration of defect structure of the type II interface in terms of grain boundary dislocations with Burgers vectors $\boldsymbol{b}^{g}$ and irrational line direction, $\xi$, (b). Equivalent descriptions of the misorientation across the interface are $\boldsymbol{n}_{s} / 2 \alpha$ and $\boldsymbol{\eta}_{1} / \pi$.

$178 \times 133 \mathrm{~mm}(300 \times 300 \mathrm{DPI})$ 


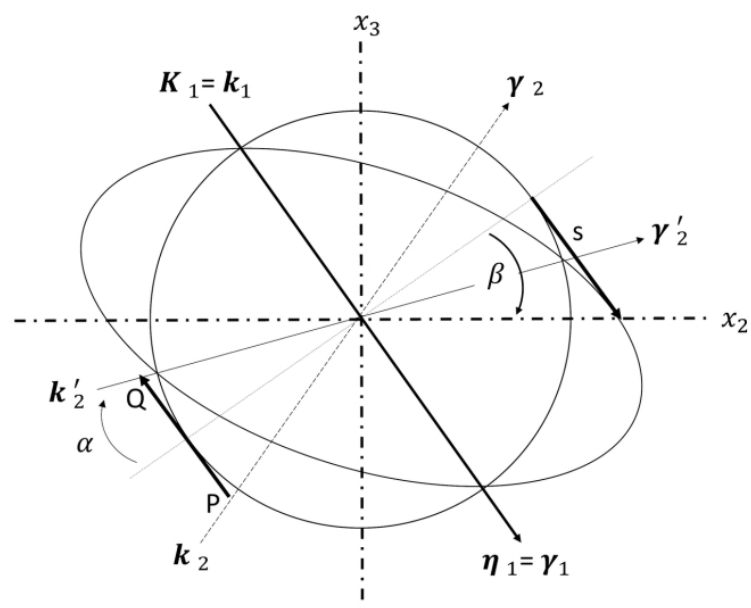

(a)

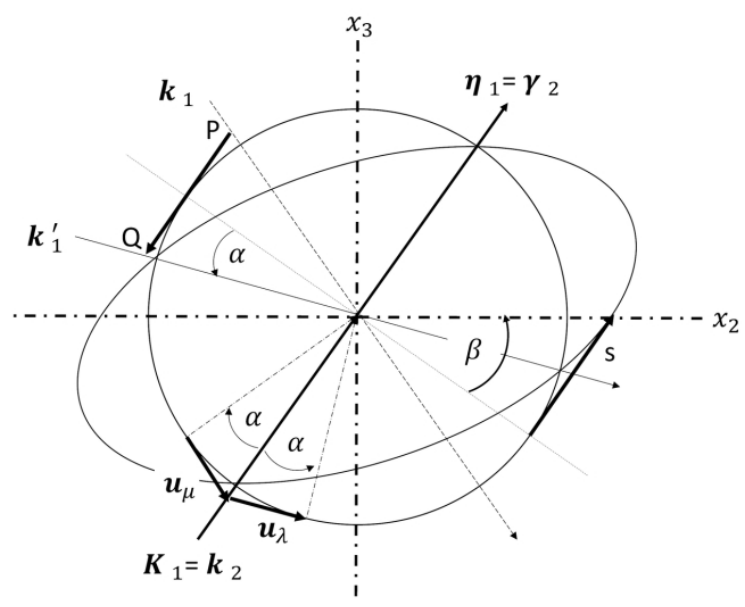

(b)

Figure A1. (a) geometrical representation of the shear, s, according to the CM for type I twins. The circle representing the undeformed state has unit radius. (b) the CM construction for type II twins. Its modification to comply with the TM is indicated by the displacements in each crystal, $\boldsymbol{u}_{\mu}$ and $\boldsymbol{u}_{\lambda}$, both with magnitude $s / 2$.

$171 \times 224 \mathrm{~mm}(300 \times 300 \mathrm{DPI})$ 


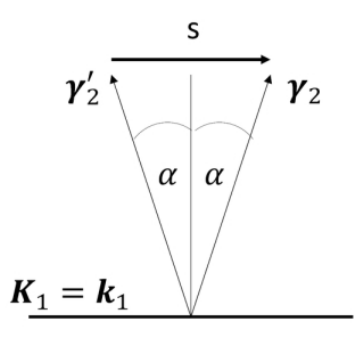

(a)

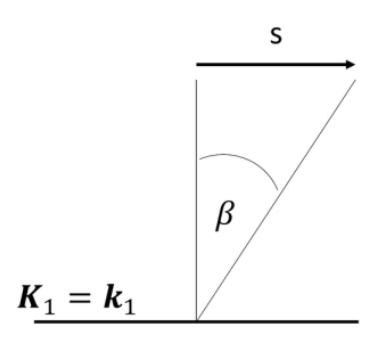

(b)

Figure A2. Mechanistic interpretation of the CM for type I twins emphasizing (a) the twin symmetry, and (b) the engineering shear.

\author{
$161 \times 71 \mathrm{~mm}(300 \times 300$ DPI $)$
}




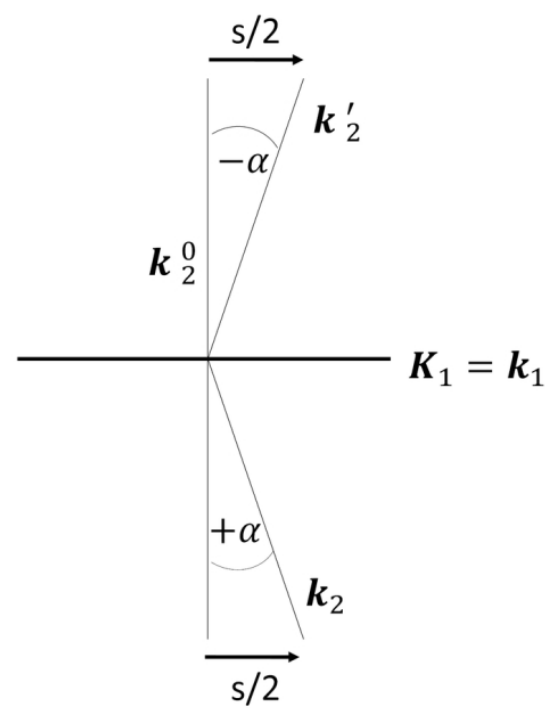

Figure A3. Hypothetical model for the formation of a type I twin involving partitioning. $140 \times 93 \mathrm{~mm}(300 \times 300 \mathrm{DPI})$ 